\title{
DETECCION DE CHLAMYDIA TRACHOMATIS EN MUESTRAS URETRALES MEDIANTE INMUNOFLUORESCENCIA DIRECTA
}

\author{
Myra Wilson Schuster"* \\ Laura Otth R.* \\ Maria Angélica Gutiérrez E." \\ Angela Tejero P." \\ Maria Isabel Moreno V."* \\ Mónica Hering
}

\begin{abstract}
WILSON Sch., M. et al. Deteccion de Chlamydia trachomatis en muestras urctrales mediante inmunofluorescencia directa. Rev. Saúde públ, S. Paulo, 23:443-6, 1989.

RESUMEN: Se estudiaron 82 pacientes con uretritis para la búsqueda de Chlamydia trachomatis mediante inmunofluorescencia directa, Neisseria gonorhoeae, Mycoplasna y Ureaplasma mediante métodos estándar. Se encontró un $19,5 \%$ de Chlamydia trachomatis y en 11 de cllos $(68,8 \%)$ se encontró asociada a otras bacterias y estos pacientes presentó una secreción escasa-gelatinosa.
\end{abstract}

DESCRIPTORES: Chlamydia Trachomatis, aislamiento. Uretra, microbiología. Inmunofluorescencia.

\section{INTRODUCCIÓN}

Las infecciones genitales por Chlamydia trachomatis constituyen actualmente un problema de salud pública en los EE.UU. y otros países, presentando una incidencia varias veces mayor que Neisseria gonorrhoeae $4,6,14,16$. En el hombre es la principal causa de uretritis no gonocócica (sobre $50 \%$ ) y de epididimitis; en la mujer es agente de infección cervical e infección pelviana aguda, y en el recién nacido de conjuntivitis y neumonia ${ }^{1,5,14,16}$.

Debido a su calidad de parásitos intracelulares obligados, requieren de cultivo celular para su aislamiento. Esta técnica es engorrosá y no está al alcance de cualquier laboratorio, razón por la cual el diagnóstico y tratamiento de las infecciones por Chlamydia se basa la mayoría de las veces en los síntomas clínicos, quedando sin tratamiento los pacientes asintomáticos ${ }^{12,21}$.

Un procedimiento alternativo, relativamente simple y rápido de realizar lo constituye la técnica con anticuerpos monoclonales teñidos con isocianato de fluoresceína. Esta metodología tiene, según la literatura, una sensibilidad que varía entre un $77,7 \%$ y un $96,3 \%$ y una especificidad que fluctúa entre 87,8 y $99,5 \% 4,11,12,20,21$.

El presente trabajo tiene por objeto determinar la frecuencia de Chlamydia trachomatis en muestras uretrales en nuestra zona, ya que no existen datos al respecto.

\section{MATERIAL Y METODO}

Se estudiaron 82 muestras uretrales de pacientes que consultaron por uretritis en el Servicio de Dermatología y Venéreas del Hospital Base de Valdivia, Chile, mediante la técnica de anticuerpos monoclonales (Micro Trak, Syva T.M.).

Las muestras se tomaron con tórula de dacrón, introduciéndola $2 \mathrm{~cm}$. por la uretra e imprimiéndole un movimiento de rotación. Luego se hizo un extendido en un área de $8 \mathrm{~mm}$. de un portaobjeto, especialmente diseñado para ello. Estos se secaron a temperatura ambiente y luego

\footnotetext{
** Financiado por la Dirección de Investigación y Desarrollo de la Universidad Austral de Chile (Proyecto S-88-19).

** Instituto de Microbiología Clínica, Universidad Austral de Chile - Casilla 567 - Valdivia, Chile.

..* Instituto de Especialidades, Universidad Austral de Chile - Casilla 567 - Valdivia, Chile.

**** Servicio de Dermatologia y Venereas. Servicio de Salud - Bueras 1003 - Valdivia, Chile.
} 
se fijaron con acetona.

La tinción se realizó con 30 microlitros de anticuerpos monoclonales y se incubó por 15 minutos en cámara húmeda. Posteriormente se lavó con agua bidestilada y se montaron con líquido especial Micro Trak.

Las láminas se observaron en microscopio de epifluorescencia con sistema de filtro para isocianato de fluoresceína, considerándose positiva toda aquella muestra que presentó 10 ó más corpúsculos esféricos que emitían fluorescencia color verde manzana brillante por extendido.

En forma paralcla se realizó cultivo para $N$. gonorrhoeae, Ureaplasma y Mycoplasma según métodos estándar ${ }^{10,15,17}$ y un frotis Gram directo para cuantificación de polimorfonucleares (PMN).

\section{RESULTADOS}

De los 82 pacientes estudiados se obtuvieron los resultados que se detallan en las Tablas 1 a 3 .

En la Tabla 1 observamos que del total de

TABLA 1

Número y porcentaje de muestras uretrales positivas y negativas para Chlamydia trachomatis mediante examen direc to con anticuerpos monoclonales.

\begin{tabular}{lcr}
\hline Muestras & No & $\%$ \\
\hline Positivas & 16 & 19,5 \\
Negativas & 66 & 80,5 \\
Total & 82 & 100,0 \\
\hline
\end{tabular}

TABLA 2

Número y porcen taje de muestras uretrales para Chlamydia trachomatis y sus asociaciones.

\begin{tabular}{lcc}
\hline Microorganismo & No & $\%$ \\
\hline Chlamydia trachomatis & 5 & 31,2 \\
Ch. trachomatis $+N$. gonorrhoeae & 5 & 31,2 \\
Ch. trachomatis + Mycoplasma sp. & 4 & 25,0 \\
Ch. trachomatis + N.gonorrhoeae & & \\
+ Mycoplasma sp. & 2 & 12,5 \\
Total & 16 & 100,0 \\
\hline
\end{tabular}

muestras estudiadas, 16 fucron positivas para Chlamydia trachontatis, lo que representa un 19,5\%.

En la Tabla 2 observamos el número y por-
TABLA 3

Relación entre microorganismos encontrados y tipo de secreción uretral.

\begin{tabular}{lccc}
\hline & \multicolumn{3}{c}{ Secrecion } \\
\cline { 2 - 4 } Microorganismo & $\begin{array}{c}\text { Franca } \\
\text { purulenta }\end{array}$ & $\begin{array}{c}\text { Escasa } \\
\text { gelatinosa }\end{array}$ & Sin \\
\cline { 2 - 4 } & No & No & No \\
\hline $\begin{array}{l}\text { Ch. trachomatis } \\
\begin{array}{l}\text { Ch. trachomatis } \\
\text { N. gonorrhoeae }\end{array}\end{array}$ & - & 4 & 1 \\
$\begin{array}{l}\text { Ch. trachomatis } \\
\text { Mycoplasma sp. }\end{array}$ & & 3 & \\
$\begin{array}{l}\text { Ch. trachomatis - } \\
\text { Mycoplasma } \\
\text { N. gonorrhoeae }\end{array}$ & 2 & 4 & \\
\begin{tabular}{l} 
Total (16) \\
\hline
\end{tabular} & $4(25,0 \%)$ & $11(68,8 \%)$ & $1(6,2 \%)$ \\
\hline
\end{tabular}

centaje de Chlamydia trachomatis y sus asociaciones, presentándose en un $31,2 \%$ de los casos sólo Chlamydia y en el $68,7 \%$ restante asociada a otros agentes de uretritis. La asociación más frecuente fue con Neisseria gonorrhoeae. No se encontró asociacion con Ureaplasma urealyticum.

En la Tabla 3 se detalla la relación entre microorganismo encontrado y el tipo de secreción. El $68,7 \%$ de los pacientes con Chlamydia presentó una secreción de tipo escasa-gelatinosa y un $25 \%$ secreción franca-purulenta, correspondiendo éstos a aquellos casos en que Chlamydia estaba asociada a $N$. gonorrhoeae.

En el $100 \%$ de las muestras se observaron más de 4 PMNs por campo microscópico en la tinción de Gram directa.

\section{DISCUSIÓN}

De los 82 pacientes estudiados $19,5 \%$ presento Chlamydia trachomatis, concordando con lo reportado en Chile por Martínez e col. ${ }^{12}$ quien obtuvo un $18 \%$ y en el extranjero con Coudron e col. $^{4}$ y Tam e col. ${ }^{20}$, quienes reportan un 18,6 y un $23 \%$, respectivamente, utilizando la misma técnica. Sin embargo, autores como Bowie e col. ${ }^{2}$, Frieberg ${ }^{7}$ y Jacobs e col. ${ }^{9}$ entre otros, relatan porcentajes que van entre un 30 y $50 \%$. Estas diferencias pueden deberse a que ellos utilizaron como técnica el cultivo celular, constituyendo 
ésta una metodología de referencia.

Por otro lado Bowie ${ }^{1}$ y McCutchan ${ }^{13}$ reportan que la frecuencia de este microorganismo es mayor en los niveles socio-económicos altos, ya que este tipo de pacientes al contar con medios económicos está más predispuesto a buscar tratamiento precozmente.

El grupo por nosotros estudiado, al igual que el de Martínez e col. ${ }^{12}$, pertenece a un grupo de nivel medio-bajo que consulta en el Policlínico de Enfermedades de Transmisión Sexual (ETS) donde la atención y tratamiento son gratuitos.

Chlamydia trachomatis se encontró asociada en el $68,7 \%$ de los casos a otros microorganismos, especialmente $N$. gonorrhoene y Mycoplasma. Este hecho plantea la necesidad de realizar en los pacientes con uretritis, un estudio microbiológico completo con el fin de disminuir la frecuencia de ETS mediante tratamicntos que se adecúen al o los microorganismos causales. No encontramos asociación de Chlamydia trachomatis con Ureaplasma urealyticum, hecho que concuerda con la literatura revisada 2,3 .
El $68,75 \%$ de los pacientes con Chlamydia trachomatis presentó una secreción escasa gelatinosa y sólo en aquellos casos en que estaba asociada a $N$. gonorrhoeae lo fue purulenta. Estos hallazgos concuerdan con la literatura ${ }^{7,8,18}$ y constituyen un buen elemento de diagnóstico presuntivo al carecer de otras metodologías que permitan establecer el diagnóstico de certeza.

Otro elemento bastante importante es que al igual que otros autores ${ }^{18,19}$ en el $100 \%$ de los casos positivos para Chlamydia trachomatis, observamos más de $4 \mathrm{PMN}$ por campo microscópico.

Como conclusión se sugiere que debido a la alta incidencia de Chlamydia trachomatis, al menos se sospeche de uretritis por este microorganismo en base al tipo de secreción, la presencia en el exudado de más de 4 PMN y a la ausencia de otros agentes de uretritis, de manera que los pacientes y sus contactos reciban tratamiento antimicrobiano adecuado con el fin de disminuir la incidencia de una de las tantas enfermedades de transmisión sexual.

WILSON Sch., M. et al. Deteç̧ão de Chlanydia trachomatis em amostras uretrais mediante imunofluorescência direta. Rev. Saúde públ., S. Paulo, 23: 443-6, 1989.

RESUMO: Em 82 doentes com uretrite foi pesquisada a presença de Chlanydia trachomatis, utilizando a prova da imunofluorescência direta, e de Neisseria gonorrhocae, Mycoplasina e Ureaplasina, utilizando os métodos padrões. Ch. trachomatis foi encontrada em $19,5 \%$ dos casos, sendo que em 11 deles $(68,8 \%)$ observou-se associação entre Chlamydia e as outras bactertias pesquisadas. Nesses pacientes observou-se presença de secreção uretral escassa e de aspecto gelatinoso.

DESCRITORES: Chlanydia trachomatis, isolamento. Uretra, microbiologia. Imunofluorescência.

WILSON Sch., M. et al. [Detection of Chlamydia trachomatis in urethral samples by means of direct immunofluorescence]. Rev. Sarúde públ., S. Paulo, 23: 443-6, 1989.

ABSTRACT: The presence of Chlamydia trachomatis was studied by the direct immunofluorescence test, as also was that of Neisseria gononhocac, Mycoplasma and Ureaplasma by the standard methods, in 82 patients with urethral discharge. Ch. trachomatis was found in $19.5 \%(16)$ of the cases and in 11 of them $(68.8 \%)$ there was association with the other bacteria investigated. This eleven patients presented a scanty gelatinous discharge.

KEYWORDS: Chlamydia trachomatis, isolation. Urethra, microbiology. Fuorescent antibody technic.

\section{REFERENCIAS BIBLIOGRÁFICAS}

1. BOWIE, W.R. Chlamydia trachomatis infections: epidemiology and therapy. Med progr., 14:51-8, 1985.

2. BOWIE, W.R.; WANG, S.P.; ALEXANDER, E.R.; FLOYD, J.; FORSYTH, P.S.; POLLOCK, H.M.; LIN,
J.L.; BUCHANAN, T.M.; HOLMES, K.K. Etiology of nongonococcal urethritis: evidence for Chlamydia trachomatis and Ureaplasma urealyticum J. clin Invest, 59:735-42, 1977. 
3. BOWIE, W.R.; POLLOCK, H.M.; FORSYTH, P.S.; FLOYD, J.F.; ALEXANDER, E.R.; WANG, S.P.; HOLMES, K.K. Bacteriology of the urethra in normal men and men with nongonococcal urethritis. J. clin. Microbiol., 6:482-8, 1977.

4. COUDRON, P.E.; FEDORKO, D.P.; DAWSON, M.S.; KAPLOWITZ, L.G.; BROOKMAN, R.R.; DALTON, H.P.; DAVIS B.A Detection of Chlamydia trachomatis in genital specimens by the Microtrak Direct Specimen Test. Amer. J. clin. Pathol., 85:89-92, 1986.

5. CHACKO, M.R. \& LOVCHIK, J.C. Chlainydia rrachomatis infections in sexually active adolescents: prevalence and risk factors. Pediatrics., 73:836-90, 1984.

6. FELMAN, Y.M. \& NIKITAS, J.A. Nongonococcal urethritis. J. Amer. med Ass., 245:381-6, 1981.

7. FRIBERG, J. Nonspecific urethritis in men. Arch. Androl 15:65-78, 1985.

8. JACOBS, N.F. \& KRAUS, S.J. Gonococcal and nongonococcal urethritis in men. Ann. intem. Med, 82:7-12, 1975 .

9. JACOBS, N.F.; ARUM, E.S.; KRAUS, S.J. Nongonococcal urethritis: the role of Chlanydia trachomatis. Ann. intem. Med, 86:313-4, 1977.

10. KELLOG, D.S.; HOLMES, K.K.; HILL, G.A. Laboratory diagnosis of gonorthoeae. In: Marcus S. \& Sherris, J.C., ed. Cumitech 4. Washington, D.C., American Society for Microbiology, 1976. p. 1-10.

11. LINDNER, L.E.; GEERLING, S.; NETTUM, J.A.; MILLER, S.L.; ALTMAN, K.H. \& WECHTER, S.R. Identification of Chlamydia in cervical smears by immunofluorescence: technic, sensitivity and specificity. Amer. J. clin. Pathol, 85:180-4, 1986.

12. MARTINEZ, M.A.; GUBELIN, W.; PINTO, M.E.; OJEDA, J.M. Diagnóstico de Chlamydia trachomais mediante examen directo con anticuerpos monoclonales. Rev. med. Chile, 113:769-71, 1985.

13. McCUTCHAN, J.A Epidemiology of venereal urethritis: comparison of gonorrheae and nongonococcal urethritis.
Rev. infect. Dis., 6:669-88, 1984.

14. PETERSON, E.M. \& MAZA, L.M. Chlamydia trachomatis: a genital pathogen worth recognizing. Focus, 48(4):247-55, 1982.

15. PRAZNICK, J. Technics for culturing Mycoplasma species from clinical specimens. Amer. J. clin Pathol, 52:784-89, 1969.

16. SANDERS, L.L.; HARRISON, H.R.; WASHINGTON, A.E. Treatment of sexually transmitted Chlamydial infections. J. Amer. med Ass., 255:1750-6, 1986.

17. SHEPARD, M.C. \& LUNCEFORD, C.D. Ureasa color test medium U9 for detection of " $T$ " Mycoplasma in clinical material. AppL MicrobioL, 20:539-43, 1970.

18. STAMM, W.E.; KOUTSKY, L.A.; BENEDETTI, J.K; LOURDEN, J.L.; BRUNHAM, R.C.; HOLMES, KK Chlamydia trachomatis urethral infections in men. Ann. intern Med, 100:47-51, 1984.

19. SWARTZ, S.L. \& KRAUS, S.J. Persistent urethral leukocytosis and asymtomatic Chlamydial urethritis. $J$. infect Dis., 140:614-7, 1979.

20. TAM, M.R.; STAMM, W.E.; HANDSFIELD, H.H.; STEPHENS, R.; KUO, Ch. Ch.; HOLMES, K.K.; DITZENBERGER, K; KRIEGER, M.; NOWINSKI, R.C. Culture independent diagnosis of Chlamydia trachomatis using monoclonal antibodies. New EngL J. Med, 310:1146-50, 1984.

21. UYEDA, Cl. T.; WELBORN, P.; ELLISON. BIRANG, N.; SHUNK, K.; TSAOUSE, B. Rapid diagnosis of Chlamydial infections with the Microtrak direct test. J. clin. Misrobiol, 20:948:50, 1984.

Recebido para publicação em 4/5/1989 Aprovado para publicação em 15/8/1989 